\title{
Mathematical Model of Hot Metal Desulfurization by Powder Injection
}

\author{
Yolanda Cepeda Rodríguez, Guillermo González Múzquiz, José Refugio Parga Torres, \\ and Luciano Eliezer Ramírez Vidaurri
}

Departamento de Metal-Mecánica, Instituto Tecnológico de Saltillo, 25280 Saltillo, COAH, Mexico

Correspondence should be addressed to Yolanda Cepeda Rodríguez, yuli_jcr@hotmail.com

Received 30 June 2011; Accepted 25 November 2011

Academic Editor: Peter Majewski

Copyright (C) 2012 Yolanda Cepeda Rodríguez et al. This is an open access article distributed under the Creative Commons Attribution License, which permits unrestricted use, distribution, and reproduction in any medium, provided the original work is properly cited.

\begin{abstract}
Although there have been a numerous number of studies on mathematical model of hot metal desulfurization by deep injection of calcium carbide, the research field as a whole is not well integrated. This paper presents a model that takes into account the kinetics, thermodynamics, and transport processes to predict the sulfur levels in the hot metal throughout a blow. The model could be utilized to assess the influence of the treatment temperature, rate of injection, gas flow rate, and initial concentration of sulfur on the desulfurization kinetics. In the second part of this paper an analysis of the industrial data for injection of calcium carbide using this model is described. From a mathematical model that describes the characteristics of a system, it is possible to predict the behavior of the variables involved in the process, resulting in savings of time and money. Discretization is realized through the finite difference method combined with interpolation in the border domain by Taylor series.
\end{abstract}

\section{Introduction}

Desulphurization of iron from the blast furnace is a wellestablished technology. There have been many studies on the desulfurization of hot metal and steel by injection of powdered agents in the literature [1-7]. The desulfurization treatments based on the injection either of mixtures of various powders or previously melted slags aim to promote partial sulfur removal in the reactor, where the reactant powder agent rises to the surface, gets dispersed in the melt, or gets attached to the bubble-metal interface.

As customers increase requirements for steel quality, plants need practices that will help them remove sulfur faster and at lower cost. One important factor in the cost of a particular process is the reagent consumption to reach the aimed at sulfur content. Among the different reagents used, calcium-carbide-based reagents and magnesium-based reagents are currently the most popular. Although many researchers have studied $\mathrm{CaC}_{2}$ desulfurization [8], there is still a need to better understand this mechanism of desulfurization.
Powder, in dense phase, is pneumatically transported and injected into the liquid metal through a submerged lance; a jet is created at the outlet of the lance that penetrates into the melt until its momentum is dissipated. In such systems gas bubbles rising through the liquid enhance mixing, promote chemical reactions, and minimize temperature and chemical inhomogeneities in the melt. Also, the stirring caused in the injection process improves the top-slag desulfurization. The bubbles forming in the liquid rise upward due to buoyancy, and the kinetic energy at the nozzle exits. A number of complex phenomena take place during the injection process which require an investigation of the kinetics, thermodynamics, transport processes, and overall process dynamics to predict the dynamic removal of sulfur from hot metal [9].

The mechanism of desulphurization with calcium carbide was first studied by Talballa et al. [10]; according to their results, calcium carbide, which is solid at the temperature of liquid iron $\left(1350^{\circ} \mathrm{C}\right)$, partially dissociates into calcium vapor and a layer of graphite. This calcium vapor then reacts with sulfur dissolved in iron to form a layer of calcium sulphide along with the graphite layer. The layers of graphite and 
calcium sulphide progressively thicken to form a barrier to calcium diffusion vapor which slows the rate. The reaction between calcium carbide and the sulfur dissolved in the hot metal is shown in

$$
\mathrm{CaC}_{2}+\mathrm{S}=\mathrm{CaS}+2 \mathrm{C}
$$

Chiang and Irons [11] showed that the reaction was controlled by the diffusion of sulfur through the particle boundary layer, rather than the diffusion of calcium vapour in the product layer. Their experimental results confirmed that the reaction could be described as a first-order, diffusioncontrolled reaction. In a further work, Irons [12] examined mixing as a possible controlling step in the desulphurization process. He concluded that mixing was always fast enough not to be rate controlling. Consequently, the reaction is controlled by diffusion through the boundary layers around the particles.

In this paper, a mathematical model is developed to obtain design guidelines and to predict the influence of the main parameters on the desulphurization efficiency of the process that will be of great help for plant engineers to improve and optimize the desulphurization with calcium carbide.

\section{Materials and Methods}

One approach to predicting the mechanism of desulfurization is to use a dimensional model, which could be dynamically updated. The model takes into account the kinetics, thermodynamics, and transport processes to predict the sulfur levels in the hot metal. A sensitivity analysis is performed to aid in the optimal selection of operating parameters. The model results allow the selection of operating conditions to minimize the processing time for desulphurization.

2.1. Cylindrical Coordinates Model. As our first step toward developing an appropriate mathematical model for such injection phenomena, it was decided to consider the case of axisymmetric gas injection into cylindrical ladle. Then, in cylindrical coordinates, the governing differential equation can be written as follows:

$$
\frac{\partial C_{A}}{\partial t}=D_{A B}\left[\frac{1}{r} \frac{\partial}{\partial r}\left(r \frac{\partial C_{A}}{\partial r}\right)\right]-\left[V_{r} \frac{\partial C_{A}}{\partial r}\right]-k C_{A} .
$$

The cylindrical model has been proposed based on the following assumptions and considerations.

(1) The reaction is described as a first-order, diffusioncontrolled reaction.

(2) There is no variation of flow properties in the $\theta$ and $z$ directions.

(3) The bubble rising is described by the radial velocity $V_{r}$.

(4) Parameters $D_{A B}$ (diffusion coefficient) and $k$ (kinetics coefficient) are temperature dependant as shown in

$$
\begin{gathered}
D_{A B}=D_{0} * e^{E a / R T}, \\
k=k_{0} * e^{E a / R T} .
\end{gathered}
$$

Initial and boundary conditions are presented in

$$
\begin{gathered}
C_{A}(r, 0)=0, \\
C_{A}\left(r_{0}, t\right)=C_{0}, \\
\frac{\partial C_{A}\left(r_{1}, t\right)}{\partial r}=0 .
\end{gathered}
$$

2.2. Spherical Coordinates Model. In order to evaluate the effect of the reactor shape, it was decided to consider the case of axisymmetric gas injection into spherical ladle. Then, in spherical coordinates, the governing differential equation is

$$
\frac{\partial C_{A}}{\partial t}=D_{A B}\left[\frac{1}{r^{2}} \frac{\partial}{\partial r}\left(r^{2} \frac{\partial C_{A}}{\partial r}\right)\right]-\left[V_{r} \frac{\partial C_{A}}{\partial r}\right]-k C_{A} .
$$

The spherical model has been proposed based on the previous considerations.

Initial and boundary conditions are presented in

$$
\begin{gathered}
C_{A}(r, 0)=0, \\
C_{A}\left(r_{0}, t\right)=C_{0}, \\
\frac{\partial C_{A}\left(r_{1}, t\right)}{\partial r}=0 .
\end{gathered}
$$

2.3. Finite Difference Method. The differential equations were discretized using finite difference method for both cylindrical (7) and spherical (8) models:

$$
\begin{aligned}
C T= & D_{A B}\left(\frac{C_{A}(r+\Delta r)-2 C_{A}(r)+C_{A}(r-\Delta r)}{h^{2}}\right) \\
& +D_{A B} \frac{1}{r}\left(\frac{C_{A}(r+\Delta r)+C_{A}(r-\Delta r)}{2 h}\right) \\
& -\left[V_{r} \frac{C_{A}(r+\Delta r)+C_{A}(r-\Delta r)}{2 h}\right]-k C_{A}, \\
C T= & D_{A B}\left(\frac{C_{A}(r+\Delta r)-2 C_{A}(r)+C_{A}(r-\Delta r)}{h^{2}}\right) \\
& +D_{A B} \frac{2}{r}\left(\frac{C_{A}(r+\Delta r)+C_{A}(r-\Delta r)}{2 h}\right) \\
& -\left[V_{r} \frac{C_{A}(r+\Delta r)+C_{A}(r-\Delta r)}{2 h}\right]-k C_{A} .
\end{aligned}
$$

The designed mesh system consists of 51 nodes for time intervals of 1 second. The group of differential equations can be solved using a fourth-order Runge-Kutta method under the initial conditions.

2.4. Main Parameters. A cylindrical vessel of $15.7 \mathrm{~m}^{3}$ and a spherical vessel of $12.6 \mathrm{~m}^{3}$ were considered. The vessel contained molten iron, at $1300-1500^{\circ} \mathrm{C}$. A stream of gas was injected vertically through an annular nozzle located centrally at the middle of the tank. The gas was injected with a uniform velocity of $100 \mathrm{~m} / \mathrm{s}$. The flow was assumed to be axisymmetric. Computations were performed in transient mode. A mesh system of 51 nodes was used (Table 1). After preliminary calculations, this mesh system was found to be a good compromise between the computational accuracy and cost. The main operation parameters are shown in Table 2. 
TABLe 1: Mesh distribution.

\begin{tabular}{|c|c|c|c|c|c|}
\hline Node & Radium [m] & Node & Radium [m] & Node & Radium (m) \\
\hline 0 & 0.1 & 17 & 0.406 & 34 & 0.712 \\
\hline 1 & 0.118 & 18 & 0.424 & 35 & 0.73 \\
\hline 2 & 0.136 & 19 & 0.442 & 36 & 0.748 \\
\hline 3 & 0.154 & 20 & 0.46 & 37 & 0.766 \\
\hline 4 & 0.172 & 21 & 0.478 & 38 & 0.784 \\
\hline 5 & 0.19 & 22 & 0.496 & 39 & 0.802 \\
\hline 6 & 0.208 & 23 & 0.514 & 40 & 0.82 \\
\hline 7 & 0.226 & 24 & 0.532 & 41 & 0.838 \\
\hline 8 & 0.244 & 25 & 0.55 & 42 & 0.856 \\
\hline 9 & 0.262 & 26 & 0.568 & 43 & 0.874 \\
\hline 10 & 0.28 & 27 & 0586 & 44 & 0.892 \\
\hline 11 & 0.298 & 28 & 0.604 & 45 & 0.91 \\
\hline 12 & 0.316 & 29 & 0.622 & 46 & 0.928 \\
\hline 13 & 0.334 & 30 & 0.64 & 47 & 0.946 \\
\hline 14 & 0.352 & 31 & 0.658 & 48 & 0.964 \\
\hline 15 & 0.37 & 32 & 0.676 & 49 & 0.982 \\
\hline 16 & 0.388 & 33 & 0.694 & 50 & 1 \\
\hline
\end{tabular}

TABLE 2: Main operation parameters.

\begin{tabular}{|c|c|c|c|c|c|}
\hline Parameter & Numerical value & Parameter & Numerical value & Parameter & Numerical value \\
\hline$D_{0}$ & $0.0001 \mathrm{~m}^{2} / \mathrm{s}$ & $K_{0}$ & $0.02 \mathrm{~s}^{-1}$ & $r$ & $0.1-1 \mathrm{~m}$ \\
\hline$E_{a}(D)$ & $0.005 \mathrm{~J} / \mathrm{mol}$ & \multirow{2}{*}{$C_{0}$} & \multirow{2}{*}{$0.01 \%$} & \multirow{2}{*}{$t_{\text {final }}$} & \multirow{2}{*}{$1800 \mathrm{~s}$} \\
\hline$E_{a}(k)$ & $0.0004 \mathrm{~J} / \mathrm{mol}$ & & & & \\
\hline$R$ & $8.314 \mathrm{~J} / \mathrm{mol} \mathrm{K}$ & $V_{r}$ & $0.001 \mathrm{~m} / \mathrm{s}$ & $\Delta t$ & $1 \mathrm{~s}$ \\
\hline$T$ & $1400^{\circ} \mathrm{C}$ & Nodes & 51 & Precision factor & 0.00001 \\
\hline
\end{tabular}

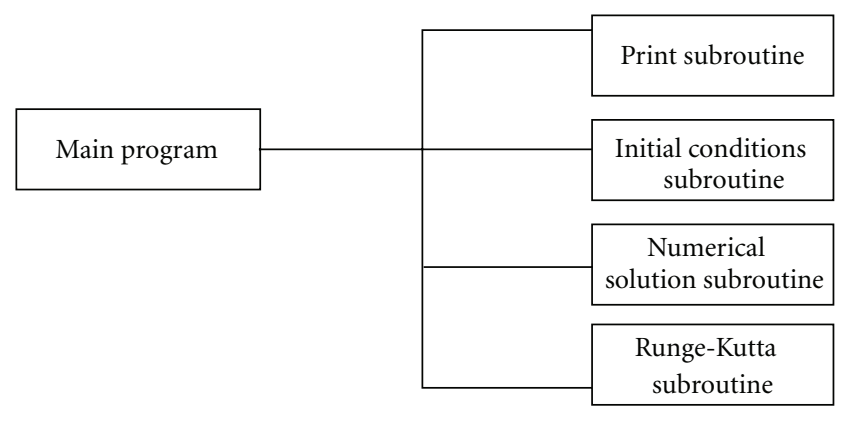

FIgURE 1: Numerical solution scheme.

2.5. Computational Solution. Both expressions of continuity equation were tested by computational methods for the model verification. The numerical solution algorithm consists of the subroutines shown in Figure 1.

\section{Model Predictions and Discussion}

3.1. Comparative Analysis between Both Schemes. Figures 2, 3,4 and 5 show the comparison between both expressions

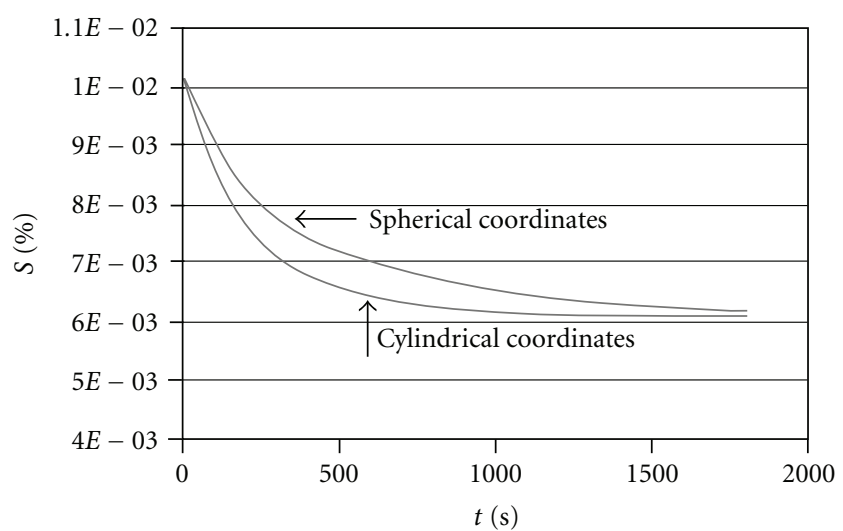

Figure 2: Sulfur elimination against time, node $10, T=1400^{\circ} \mathrm{C}$.

of continuity equation: cylindrical coordinates and spherical coordinates for desulfurization with calcium carbide injection. One can see from Figures 2, 3, 4 and 5 that the cylindrical model agrees fairly well with the sphericalmodel.

The desulphurization rate of the cylindrical model is greater than that of the spherical model especially during the calcium carbide injection, although both schemes have 


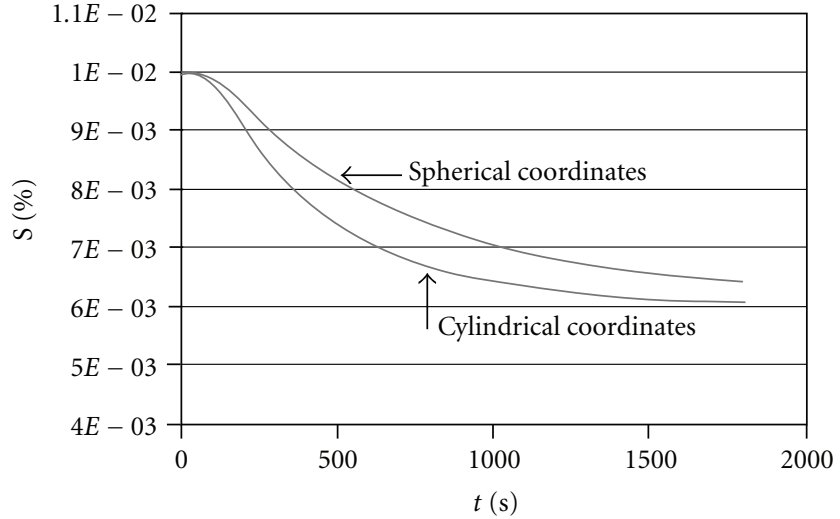

Figure 3: Sulfur elimination against time, node $20, T=1400^{\circ} \mathrm{C}$.

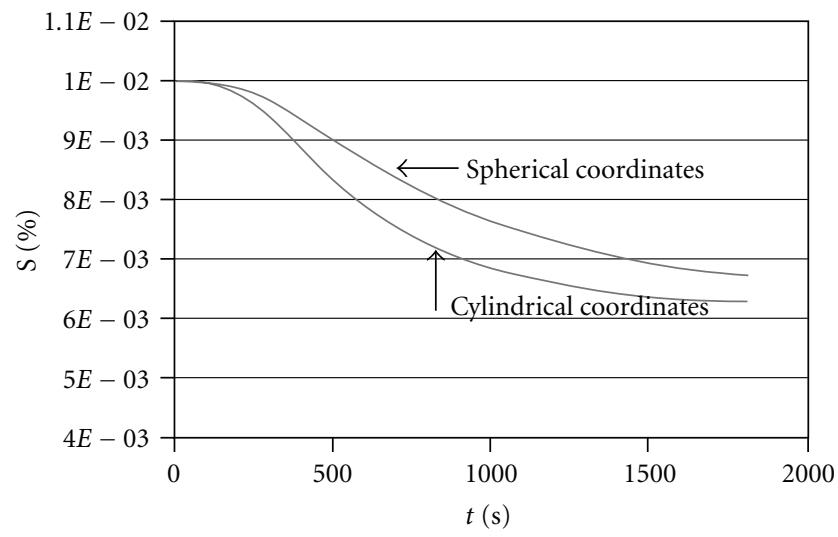

Figure 4: Sulfur elimination against time, node $30, T=1400^{\circ} \mathrm{C}$.

practically the same incubation period. The process reaches almost equilibrium at the end of injection.

3.2. Comparative Analysis with Industrial Data. Numerical model was also tested using experimental data reported by other investigators. Enríquez et al. [13] showed that an adequate control of harmful elements in steel fabrication is only reached by sulfur levels under 0.005 and it is feasible in the practice to achieve sulfur levels of 0.001 .

Rellermeyer et al. [14] implemented a treatment series that indicates initial sulfur level between 0.02 and 0.06 and final sulfur level between 0.005 and 0.01 .

Freissmuth et al. [15] showed a sulfur profile with initial values between 0.01 and 0.04 and final values between 0.007 and 0.01 with a process duration of 8 and 14 minutes.

Meichner et al. [16] found initial sulfur levels between 0.01 and 0.03 and final sulfur levels between 0.002 and 0.008 in a time of approximately 10 minutes.

Figueroa [17] found sulfur elimination percents between 37 and $68 \%$ with process duration of around 10 minutes.

The proposed model reaches an elimination percent of $40 \%$ with an initial sulfur concentration of 0.01 and a final sulfur concentration of 0.005 . The efficient process duration is around 13 minutes. One can see from the industrial data that the model prediction agrees fairly well with the practical results.

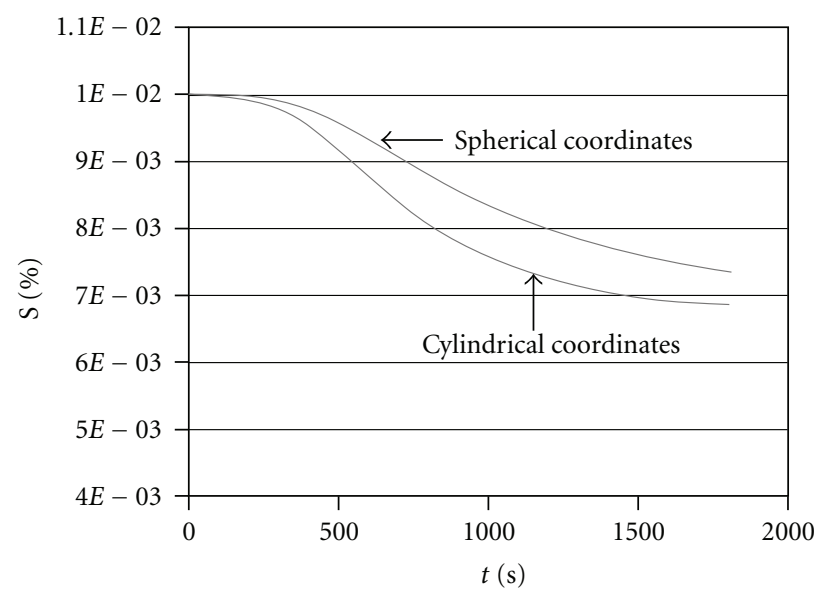

FIgUre 5: Sulfur elimination against time, node $40, T=1400^{\circ} \mathrm{C}$.

The industrial data shows that for the process of desulphurization using $\mathrm{CaC}_{2}$ it is possible to obtain approximately a $49.69 \%$ of sulfur elimination in a time range of $10-25$ minutes. The temperatures in both cases are around $1400^{\circ} \mathrm{C}$ and the elimination profiles are very alike.

\section{Conclusions}

Based on the analyses of thermodynamics and kinetics, a mathematical model has been developed, particularly with the three basic parameters being taken into account, to simulate the variation of sulfur in hot metal with time. Model verification and simulation analyses were carried out, arriving at the following main conclusions.

(1) The cylindrical model agrees fairly well with the spherical model.

(2) The model prediction agrees well with the practical results.

(3) The process reaches equilibrium mainly at $1500 \mathrm{sec}-$ onds and has an incubation period of 150 seconds approximately.

\section{Nomenclature}

$C_{A}$ : Sulfur concentration

$D_{A B}$ : Diffusion coefficient

$k$ : Kinetics coefficient

$r$ : Variation coordinate

$t$ : Time

$V_{r}:$ Radial velocity

$D_{0}$ : Initial diffusion coefficient

$K_{0}$ : Initial kinetics coefficient

$E_{a}$ : Activation energy

$R: \quad$ Gas constant

T: Temperature

$r_{0}:$ Internal radium

$r_{1}:$ External radium

$C_{0}:$ Initial concentration

$C T$ : Concentration derivative discretization

$h$ : Spatial coordinate discretization. 


\section{Acknowledgment}

The authors would like to thank the Departamento de Metal-Mecánica of the Instituto Tecnológico de Saltillo for supporting this paper.

\section{References}

[1] C. F. Landefeld and S. Katz, "Kinetics of iron desulfurization by $\mathrm{CaO}-\mathrm{CaF}_{2}$," in Proceedings of the Process Technology, vol. 6, pp. 429-439, 1986.

[2] S. Ohguchi, D. G. Robertson, B. Deo, P. Grieveson, and J. H. E. Jeffes, "Simultaneous desulphurization and dephosphorisation of molten pig iron," Ironmaking and Steelmaking, vol. 11, pp. 202-213, 1994.

[3] D. G. Robertson, S. Ohguchi, and A. Deoand, "Theoretical and laboratory studies of injection phenomena," in Proceedings of the Scaninject III, vol. 8, pp. 1-8, SCANINJECT, Lulea, Sweden, June, 1983, Part 1, Mefos.

[4] P. Grieveson and B. Deo, "Desulfurization of molten pig iron containing aluminum by powder injection," Steel Research, vol. 59, pp. 263-268, 1988.

[5] P. Grieveson, S. B. Deoand, and S. Ohguchi, "Multicomponent mixed transport control theory for the kinetics of coupled slag-metal and slag-metal-gas reactions," Ironmaking and Steelmaking, vol. 11, pp. 41-56, 1984.

[6] P. Grieveson and B. Deo, "Desulphurization of molten pig iron containing aluminum by powder injection," Steel Research, vol. 59, pp. 263-268, 1988.

[7] P. Grieveson and B. Deo, "Kinetics of desulphurization of molten pig iron," Steel Research, vol. 57, pp. 514-519, 1986.

[8] G. A. Irons, "The role of mixing in powder injection desulfurization processes," Ironmaking and Steelmaking, vol. 16, pp. 28-36, 1989.

[9] A. Cameron, "External treatment of hot metal," in Blast Furnace lronmaking, Lecture Notes, chapter 17, p. 17, McMaster University, Hamilton, Canada, 1992.

[10] M. Talballa, K. Tojan, and L. O. Brockway, "Mechanism of desulphurisation of liquid iron carbon alloy with solid $\mathrm{CaC} 2$ and CaO," AFSTrans, vol. 775, pp. 76-122, 1976.

[11] L.-K. Chiang and G. Irons, "Kinetic studies of calcium carbide hot metal desulphurisation by powder injection," ISS Transactions, vol. 1, p. 36, 1991.

[12] G. A. Irons, Bubble formation at nozzles in pig iron, Ph.D. thesis, McGill University, Toronto, Canada, 1978.

[13] J. L. Enríquez and E. Tremps, Metalurgia Secundaria, Monografías sobre Tecnología del Acero. Parte II, 2007.

[14] H. Rellermeyer, W. Meichner, and W. Gmohling, "Method Process and Composition for Desulfurizing Pig-Iron Melts," US Patent, 1984.

[15] A. Freissmuth, W. Heinl, H. Knahl, E. Pfluger, and J. Schokenbroek, "Desulfurization Agent and Process," US Patent, 1998.

[16] W. Meichner, W. Gmohling, M. Tutte, and K. Peters, "Fine Grained Agent for Desulfurizing Molten Iron,” US Patent, 1996.

[17] A. Figueroa, "Desulfuración externa de arrabio," Reporte Centro de Graduados Tecnológico de Morelia, 1994. 

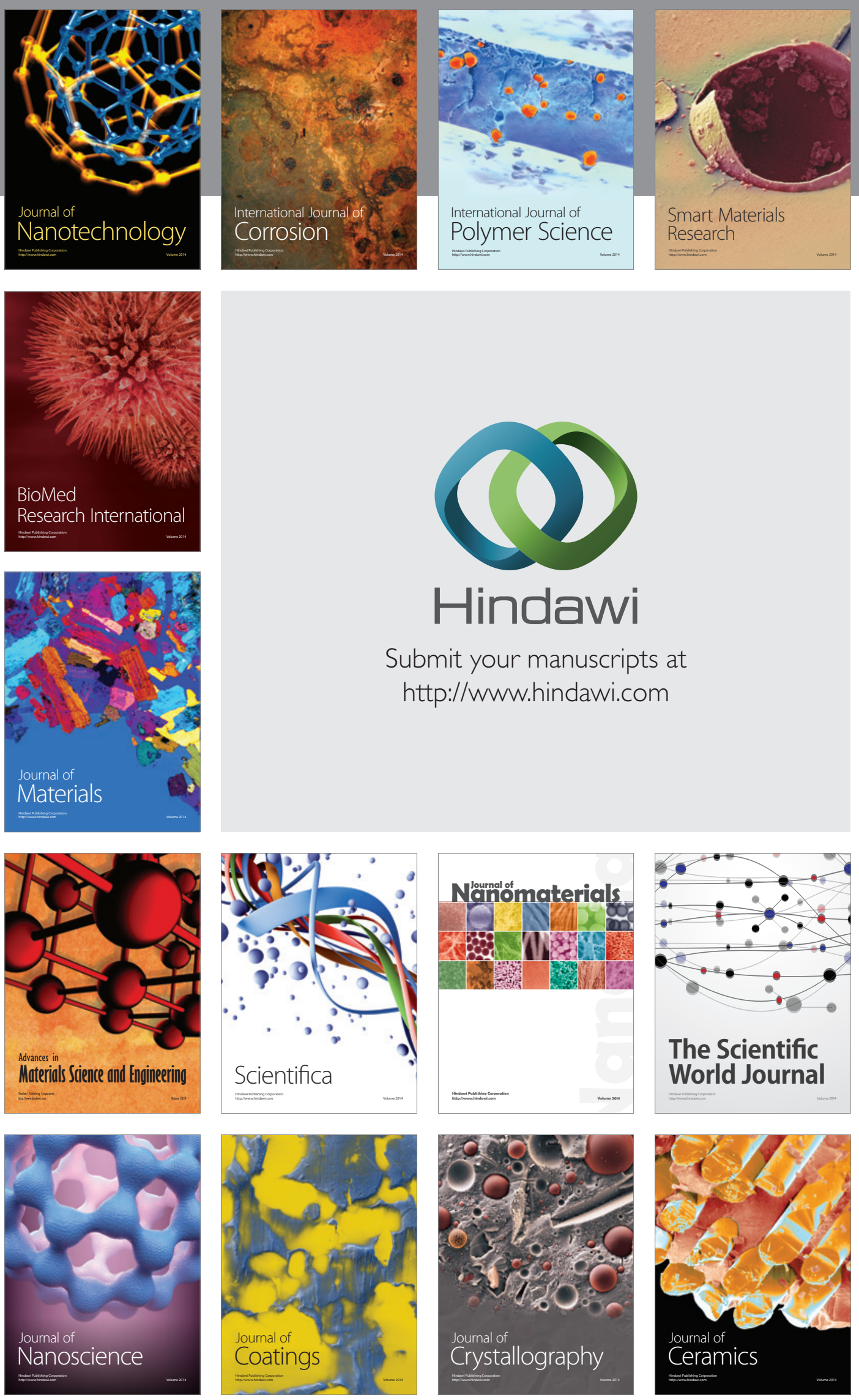

The Scientific World Journal

Submit your manuscripts at

http://www.hindawi.com

\section{World Journal}

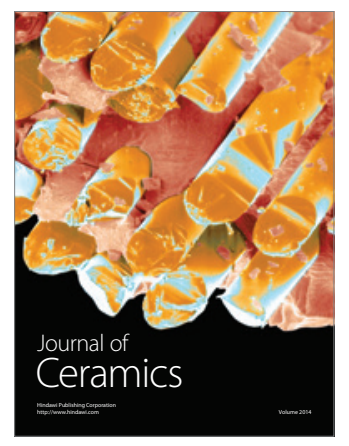

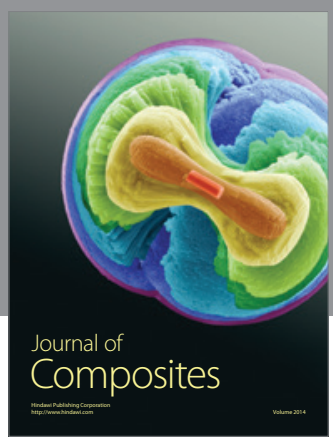
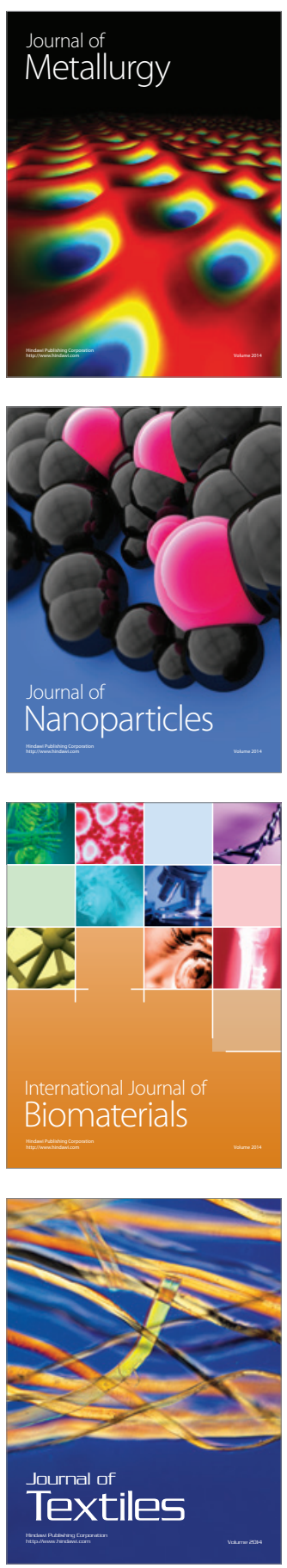